\title{
Maintaining a minimassive database of microdrinking measures
}

\author{
HOWARD L. KAPLAN \\ Addiction Research Foundation of Ontario, Toronto, Ontario, Canada
}

\begin{abstract}
For longitudinal studies of rats' consumption of ethanol, water, and food, we require manual entry of directly observed measurements, consolidation with computer-collected measurements, quick computation of derived statistics, and the export of selected data for further analysis and graphing. As in a spreadsheet, our solution propagates changes in data values through all computations dependent on them but, as in a relational database, maintains data in discrete disk records indexed by day and cage, in which the format of these records is also described by records stored in the same database. During manual data updates, each day's data are presented in a spreadsheet-like format but with more control over data entry validity than a spreadsheet typically affords. Experimental integrity is further enhanced by producing printed worksheets for data entry, assignment of rats to treatments, and printed injection instructions directly from such customized data files.
\end{abstract}

We are currently studying the effects of drugs on rats' microdrinking of ethanol and water-not only how much they consume, but also at what times, in what size drinks, with what regularity, and so forth. In a system that we call a drinkometer, pressure transducers are used to record fluid volumes remaining at 1 -min intervals, and changes in this value are stored on disk files by a laboratory computer running under MS-DOS; the summary microdrinking statistics are extracted later. In addition to these drinkometer detail files, we also record manually read data, including rat body weights, starting and ending fluid volumes, and food weights.

Several features make our situation unusual:

1. Experiments are not so much planned as planted, as one would do in a garden-harvests are variable as to time and amount. At best, only half of the rats entered into an experiment learn to drink ethanol, and the time required to do so may depend on factors beyond our control. Therefore, we require daily progress reports of statistics (such as grams of ethanol consumed per kilogram of body weight) computed from data that are less than 1 day old; we use these statistics both to monitor our training and to assign rats to blocks and treatments.

2. Because of the small numbers of subjects in our studies, we cannot assume that data-recording errors will average out in the long run, and it is worth extra effort to ensure data accuracy; therefore, duplicate data entry (i.e., keying verification) is required.

3. Interim statistical results are often required within a few days of an experiment's conclusion, so that a subsequent experiment can be initiated and the limited number of pressure transducers kept profitably occupied.

Correspondence should be addressed to the author at the Addiction Research Foundation, 33 Russell Street, Toronto, Ontario M5S 2S1, Canada.
In the context of this session, which consists of developing strategies for dealing with massive databases, this work shares several attributes with these databases: (1) It is too large for managing well by spreadsheet. (2) The studies are inherently longitudinal, with data entered during the studies rather than at their end. (3) The list of measures collected changes between, and sometimes grows during, experiments. (4) The amount of data is large enough for computer-performance issues to be relevant.

\section{Limitations of Standard Software}

In some ways, what we want resembles what we could get from a spreadsheet: data organized by tables, with derived statistics (e.g., g/kg consumption) computed as quickly as the data can be entered. However, a typical experiment involves an initial population of 60 rats, of whom about half will continue for $\mathbf{4 0}$ days, with at least 11 manually entered and up to 50 derived measures per rat. The product of 60 rats, 40 days, and 60 measures is 144,000 items, which is a large amount of data to try to organize as a set of spreadsheets, even if enough memory is available. Furthermore, the data are inherently three-dimensional (rats, days, and measures), and even if linked spreadsheets are used to partition the data along one dimension, most spreadsheets are adept at displaying slices in only one set of parallel planes. More critically, if the spreadsheet is to update computed measures, then its contents would not be only numbers but also computational formulas for derived variables; it seems inappropriate to duplicate this structural information by copying formulas across rats when the data should be brought, case by case, to the formulas. Such data movement can be accomplished by spreadsheet macros, but the performance penalties are too great in our application. In addition, spreadsheet macro languages lack most of the code- 
and data-structuring features considered desirable for large programming projects. One last, important disadvantage of spreadsheets for this kind of data management is the lack of a specific value to represent missing data and to propagate appropriately through all formulas.

Let me note, incidentally, that we have found that a spreadsheet is quite valuable at another stage of our experiments, the production of final tables and graphs. In particular, we use SAS to produce summary ASCII files, which we then pass to Quattro Pro, which can combine high-quality graphs and summary ANOVA tables onto single pages, producing a legible and compact report of our analyses. It is because this task is primarily one of page management, rather than data management, that the spreadsheet is an appropriate tool.

For data management, however, we found many of our required features in database software: data organized as one record per rat per day, with the various measures as fields along the length of the record, and random access to any day's data, as required. The separation of the storage of data and code, a separation that does not occur in a spreadsheet, makes it convenient to develop new code for analysis and reports without any problem in retrofitting the code into the structure of an existing spreadsheet; this is important when experiments must begin while critical software features are still under development. Although some databases do allow the definition of calculated fields that are derived from other data in the same record, we need the ability to calculate quickly across the day dimension, instead of the measure dimension, to get statistics such as weight gain; we also need the ability to access the drinkometer detail file, which is external to the database, and to compute summary statistics dependent on both the database and the external data.

Any database or spreadsheet that we would use, however, would need some way to process the raw drinkometer data records, the minute-by-minute data. Each drinkometer file consists of a variable number of variable-length records, one record per drinking tube, containing pairs of minute numbers and corresponding transducer voltages. This is the kind of data that is processed much more efficiently by a compiled, numeric-intensive language such as Pascal, FORTRAN, or $\mathrm{C}$ than by the programming language of a typical database or by spreadsheet macros. However, the processing cannot be independent of the manual data, because the manually read starting and ending fluid levels are used as calibration information in extracting microdrinking statistics. Therefore, whatever database is used must either have a very efficient data-manipulation language or else link easily to external programs.

This need to process raw drinkometer files is one of the reasons why we do not use SAS, which has some databaselike features, for our initial data management but only for subsequent statistical computations: SAS does not have convenient links to external programs. Another major reason is that, for the complexity of the operation we require, the development and the debugging environment of SAS are not adequate. Moreover, SAS is perceptibly slower than we would like on the 80286-based computer on which we run it; this deficiency, but not the others, could be solved by purchasing a faster computer, a solution that would be much less costly than the development time we have spent instead.

\section{A Home-Built Solution}

Because no commercially available spreadsheet, database, or statistical package seemed to have enough of the features we needed, I decided to develop this system by using the compiler Turbo Pascal 5.0 (Borland) and the subroutines library Turbo Professional (Turbo Power Tools). The resulting software consists primarily of two programs, DRINKMTR and DATAEDIT. DRINKMTR, which always executes in conjunction with DATAEDIT, is much smaller and simpler; it is limited to reading the drinkometer transducers, storing the minute-by-minute results on disk, and exchanging status information with DATAEDIT. The primary reason for making it a separate program is that the interrupt-processing routines necessary for scheduling tube readings interfere with the debugging tools needed to develop the remainder of the software. DATAEDIT contains all of the other features discussed in this paper; to implement them required the in-house development of two major utility packages: a data-entry screen manager and a singly indexed keyed-file manager.

The screen manager does not use the screen-painting approach that is common in many commercially advertised packages. Instead, forms are generated at run time from tables of definitions created as part of the programs. This is similar to the approach taken by relational databases such as Paradox or Oracle, in which a usable, if not optimal, screen can be automatically generated to view any table (a set of similarly structured records). In such forms, records are rows, fields are columns, and the labels and formats are considered part of the data definition rather than the screen definition. In the context of the drinkometer laboratory, the measures to be included in any experiment are defined as rows of a table of measures, in which the columns are attributes such as column headers, eight-character formal names, ranges, number of digits and decimal places, and computing formulas. These measures subsequently define columns in a table that has rats as rows and that is used for data entry and for storage of daily results. The screen manager also uses the same definitions to format data for printed reports and for ASCII files output for other programs.

Both structural information (such as variable definitions) and experimental data are stored as records in a heterogeneous, singly indexed file. Each record key begins with a letter specifying the record type: For example, "D1003007" is a data record for Phase 1, Day 3, Cage 7; "Y1004"' is a global record for Phase 1, Day 4. Names beginning with "@" and a letter contain the definitions of records whose names begin with that letter, so, for example, the series of records beginning with "@Y" defines the structure of the global day records, and record names beginning with “@D” contain the definitions of 
manual and computed measures stored on the daily data records. This database structure allows the easy generation of table loaders, browsers, and other structured tools. The philosophy behind this arrangement is similar to that of a relational database: Any one table consists of homogeneous records, and all data are stored in such tables, including data about the structure of the database. Although in a true relational database, all of the operations on tables yield other tables as results, in our database, many of the operations we have defined work at the record level rather than at the table level.

This database structure achieves acceptably fast performance because it is limited to one key and a maximum file size of several thousand records; this allows the entire index to be kept in memory while the file is open, so only one disk access is needed to retrieve or rewrite a record, and only two are needed to create or delete one. Records are stored in fixed-length slots and are limited to 510 bytes (plus 2 bytes overhead), so that no record spans a disk sector; since much of this space can be wasted in experiments with short sets of measures, files are automatically compressed for daily backup to diskette. It should be noted that the drinkometer detail files are kept separately, one file per day; among other reasons for this is that a combined data file would quickly grow too large for efficient backup.

The definitions stored on the database allow the datacollection and data-entry processes to be tailored to each experiment. Daily measures are divided into three classes: carryforward measures (e.g., the identity of a rat occupying a given cage), which rarely change from one day to the next; manual measures, which must be collected every day; and derived measures, which are computed from carryforward and manual measures. To reduce the opportunities for data-recording errors, the forms on which the raw data are recorded are themselves generated from each experiment's database, with the column labels corresponding to the carryforward and manual measures to be collected, and with the last available day's data already entered into the carryforward columns. At data-entry time, carryforward measures' data are automatically initialized from the previous day's values, so that only the rare exceptions need to be keyed.

\section{Data Integrity}

One feature of DATAEDIT that may be uncommon in laboratories is the provision for duplicate data entry. All manually measured statistics are entered twice by independent research assistants from paper-recording forms onto separate copies of the input screen. Only when both are done are the two entries compared and the (usually small) number of discrepancies resolved. Prior to this resolution, the second assistant's entries occupy the space reserved for the computed statistics; thus, no statistics can be computed until the data are verified as having been entered correctly. After that point, further corrections can still be made, but only a single entry is required. DATAEDIT does not attempt to prevent fraud by high-security features such as password-protected access to the data file; rather, it provides trusted laboratory staff with the tools to reduce honest error.

Another important data-integrity feature is the maintenance of the computed statistics when the inputs or formulas change. Let us consider two cases, one statistic that depends on two days' data and another that depends on the external file of minute-by-minute records; their formulas would look like this:

$$
\begin{gathered}
\text { WGAIN = NEXT WEIGHT - WEIGHT } \\
\text { DRINKMAX = \$PCTVOL(ETUBE, 1,720,5) }
\end{gathered}
$$

The first formula computes weight gain from the current day's and next day's weight; the second calls a function to determine the time at which $5 \%$ of the fluid in the ethanol tube has been consumed. This function takes four parameters: the tube number, the time span to be considered, and the percentage of consumption to be measured. These formulas are entered and parsed at the time variables are defined, and their translations are stored as part of the measure definitions. At that time, each formula's dependencies are also calculated: The first formula depends on the weight measure on both the current day and the subsequent day, and the second depends on the ethanol-tube number and (implicitly) the contents of the drinkometer detail file. Any program operation that can change items on which formulas depend includes, as part of its code, a call to procedures that propagate the change through only the affected records. For example, when data for Day 17 of an experiment have been entered and verified, the program automatically updates the weight-gain measure on Day 16 for all rats who are still present on Day 17; prior to that, the Day 16 weight-gain measure was set to "missing." On the other hand, if only one rat's ethanol-tube number had been incorrectly entered and subsequently changed, only that one rat's record for that day would be updated for all measures dependent on the tube number. The use of the dependency rules to provide a minimal recalculation is another feature that DATAEDIT has borrowed from the spreadsheet world.

Because the scope of DATAEDIT is limited to data integrity and experimental management, very few summary statistics (statistics averaged across days or across rats) are generated or produced within the program. The one important exception occurs when rats are blocked for assignment to treatment. At that time, the program computes the means and standard deviations of the baseline data for (usually) 4 days preceding the first scheduled active treatment day for one primary statistic and up to four auxiliary ones. The primary statistic is the one on which blocking is to be based, usually $\mathrm{g} / \mathrm{kg}$ ethanol consumption. The secondary statistics are ones that are not directly used in blocking but might disqualify rats from further treatment. Because we alternate the sides of ethanol and water presentation daily, we usually use the rat's percentage of total fluid consumed from the ethanol tube and from the left-side tube as secondary statistics. If any rat's prefer- 
ence is stronger for the left or right side than it is for ethanol (as assessed by the means and standard deviations of the two statistics), we do not enter the rat into further treatment, regardless of how high the mean daily ethanol consumption is.

The process of selecting rats for assignment to treatment is a semi-interactive one. The operator selects the critical statistics, DATAEDIT sorts the rats by those statistics, the operator marks the ones eligible for blocking, DATAEDIT presents a tentative assignment to blocks, the operator may make modifications (e.g., to redistribute the rats among complete and incomplete blocks), DATAEDIT presents a tentative assignment to treatments, and the operator may override these assignments.

Once the treatment assignments have been stored on disk, they are subsequently used in the preparation of daily injection planning charts. The charts specify not only which drug a rat is to receive but what volume of solution is required to administer a dose scaled to the rat's body weight. In our typical Latin-square design, each treatment name is a sequence of four letters representing four alternative drug doses. When the operator specifies which treatment cycle is to be included in the injection chart, the program chooses the appropriate letter from each rat's dose sequence and prints a form that specifies the cage and rat numbers, the sequence assignment, the name of the corresponding dose, the rat's weight, and the volume of solution to be injected based on that weight.

Except for the sorting of rats for blocking and assignment to treatments, no comparison or summary of rats is attempted within DATAEDIT. Instead, all of the data are exported to SAS for further computation of descriptive and inferential statistics. Because all data items have associated definitions, the skeleton SAS code to read the file is also exported at the same time as the data. If the experiment is basically the same structure as a previous one (typically, Latin-square assignment of 2-day drug treatment periods separated by 2 -day washouts), only a few minutes' manual work is required to specify the necessary analyses.

\section{Costs and Benefits}

Having discussed the benefits of DATAEDIT, I should balance that with some estimate of its costs. I do not have exact records, but I estimate that by the time it is completely developed and documented, it will have consumed half of my working hours for one year. The source code, including comments, occupies about $850 \mathrm{~K}$ of disk space; when compressed with the use of the standard PKZIP utility, it still occupies $250 \mathrm{~K}$.

The executable files for DATAEDIT consist of a $200 \mathrm{~K}$ .EXE file and a $100 \mathrm{~K}$ overlay file; DRINKMTR.EXE is an additional 30K. For large experiments, the programs require most of the $640 \mathrm{~K}$ conventional memory not occupied by MS-DOS. The programs can be run on an 8-MHz 8088based computer but are easier to use on a 10-MHz 80286based computer in which additional EMS RAM is used as a disk cache.

If we ignore the complications introduced by the drinkometer detail records, the scale of any one experiment's manual data is not much different from that found in many laboratories. For any one such experiment, the overhead involved in developing this software would not have a reasonable payoff. However, the similarities among the experiments do justify such development, providing that the software is designed to also accommodate their differences. By allowing the software to collect data definitions before it collects data, and by reusing those definitions for a variety of screen and hard-copy formats, we have incorporated such efficiency and flexibility into our datamanagement system. 